\title{
Essential Oils and their Antimicrobial Activity
}

\section{Vivian Tullio}

Department of Public Health and Pediatrics, Microbiology Division, University of Torino, Via Giuseppe Verdi, 8, Torino, Italy

\begin{abstract}
In the last twenty years, the clinical interest in "natural" medicine has greatly increased and, above all, there has been a broad use of plant products in microbiological field. Among new therapeutic approaches based on medicinal plants and their extracts, the use of essential oils is attractive and increasingly under investigation. Interest in essential oils has revived in recent decades with the popularity of aromatherapy, a branch of alternative medicine that claims that essential oils and other aromatic compounds have curative properties. Essential oils are a concentrated hydrophobic liquid containing volatile aroma compounds from of plant secondary metabolism, obtained by distillation. They are also known as volatile oils, ethereal oils, aetherolea, or simply as the oil of the plant from which they were extracted, such as "thyme red oil". Essential oils have a wide application in folk medicine, fragrance industries, as well as food flavoring and preservation, but only in recent years, they have started to be recognized for their potential antimicrobial role. The literature data report many evidence that a larger number of plants and their constituents could show beneficial therapeutic effects, including antioxidant, anti-inflammatory, antitumor, and immunomodulatory activity, which still need to be further investigated.
\end{abstract}

This new trend is due both on the increasing spread of microorganisms resistant to conventional antimicrobial agents, andto the increased studies about antimicrobial activity of essential oils. Drug resistance can arise because the bacteria and yeasts may "mutate" rapidly, changing cellular membrane proteins to no longer be recognizable by drugs. In fact, most bacteria are able to modify PBPs (a group of proteins that are characterized by their affinity for and binding of $\beta$-lactam drugs)or produce, for defensive purposes, several enzymes, such as endo and eso- $\beta$-lactamasesor transferase, able to inactivate, respectively, $\beta$-lactam and aminoglycosides antibiotics. In yeasts, the resistance to antifungal agents, especially to azoles, can arise because of changes to the efflux pumps or drastic reduction (50\%) of ergosterol level in cellular membranes, which can no longer be the drugs target site.

In addition to these problems, antibiotics used against pathogen microorganisms could have (induce) severe side collateral effects, especially in patients undergoing prolonged therapeutic treatment, or may alter the microbiome, so important for the intestinal and the entire body eubiosis. Antimicrobial agents, increasing dysbiosis, create an ideal environment for pathogen microorganism colonization and further infections with possible recurrent episodes. Conversely, essential oils, containing hundreds of naturally active ingredients in variable proportion, eliminate the risk of antibiotic resistance, since microbes are not able to adapt to their heterogeneous structure.For this reason, today, the essential oils are also tested to assess their possible clinical use.

From literature data, although fragmentary and incomplete, there is evidence that essential oils are active against many microorganisms, such as bacteria, fungi, parasites, and viruses, being able to eliminate pathogens while preserving "friendly" microorganisms. Moreover, essential oils will not harm the body, do not accumulatein the liver or kidneys, do not cause resistance, andstimulates the immune system; hence,theycould be used both to prevent and to treat microbial infections.

\author{
Publication History: \\ Received: December 20, 2015 \\ Accepted: February 28, 2016 \\ Published: March 03, 2016

\section{Keywords:} \\ Essential oils, Antimicrobial, Drugs target, Diagnosis, \\ Immunomodulatory
}

However, the promising clinical microbiology data are often insufficient, and not based on validated methodologies and experimental models with good predictability for clinical use. In this context, it is important and necessary to introduce standardized methods for the in vitro and in vivo essential oilsactivity evaluation, as for conventional drugs. The essential oils therapeutic use must be accompanied by a valid scientific investigation with results that confirm the suggested therapeutic activity. We have to remember thatessential oils, while giving fewer side effects, are not harmless if used incorrectly. To make a valid assessment of antimicrobial activity of essential oilsand an effective comparison with conventional drugs, it is important to analyze classic microbiological parameters, such as the minimal inhibitory concentration (MIC), the bactericide/ fungicide concentration (MBC/MFC), the post-antibiotic effect (PAE), the antibiogram/aromatogram, etc.

Lastly, clinical experience showed that the therapeutic efficacy of conventional antimicrobial agents depends bothon their direct effect on a given microorganism and on the activity of the host immune system.To eradicate the infectious agent it is important to evaluate even for essential oils their possible influence on host defense mechanisms to find compounds that are able to stimulate and not to interferenegatively with them.

Our research group is responsible for many years of the in vitro activity of antimicrobial drugs against clinical and environmental bacteria and fungi (yeasts and filamentous fungi) strains, and the influence of these drugs on the healthy and immunocompromised host's innate immune system. It follows that we evaluated, with the same scientific approach used for conventional drugs, both activity and the influence on the immune system of many essential oils (e.g., pine, thyme, cloves, lavender, lemon balm, sage, fennel, and others) and their components (thymol, carvacrol, eugenol, etc.) against yeasts (Candida spp. and not Candida spp.) and filamentous fungi, comparing themwiththose observed for the leading therapeutic antifungal drugs.

"Corresponding Author: Prof. Vivian Tullio, Department of Public Health and Pediatrics, Microbiology Division, University of Torino, Via Giuseppe Verdi, 8 , Torino, Italy; E-mail: vivian.tullio@unito.it

Citation: Tullio V (2016) Essential Oils and their Antimicrobial Activity. Int J Clin Med Microbiol 1: 101. doi: http://dx.doi.org/10.15344/IJCMM/2016/110

Copyright: (c) 2016 Tullio. This is an open-access article distributed under the terms of the Creative Commons Attribution License, which permits unrestricted use, distribution, and reproduction in any medium, provided the original author and source are credited. 
Our results revealed a real effectiveness of essential oils and, in particular, of some of them, to inhibit the growth of yeast and filamentous fungi (dermatophytes, Fusarium spp.), even at low concentrations. This is the case of red thyme essential oil (carvacrolchemotype), pine, clove andoregano that have proven to be the most effective, showing MIC values in some cases very low, especially for C. krusei and C. glabrata. These data are of great importance in the light of the evident drug resistance that many strains of C. glabrata and C. krusei, naturally resistant to fluconazole, showed against azoles.

It is not surprising that thyme red, cloves and oregano essential oilsshowed high fungal growthinhibition, as their main components are phenols such as thymol, carvacrol, and eugenol, compounds characterized by high bioactivity.

Pine essentialoil showed, surprisingly, an inhibitory activity higher than thyme, cloves, oregano towards some strains of Candida spp.; its main components are terpene hydrocarbons which, as many studies have already demonstrated, possess antimicrobial capabilities against fungi, bacteria and viruses, proving to be one of the classes of compounds of plant origin most active in this regard.

To evaluate the action of essential oilson the immune system, human PMN against C. albicans (thyme-sensitive) and C. krusei resistant to fluconazole and anidulafungin assessed the red thyme essential oil influence, at sub MIC/MIC concentrations, on the activity of intracellular killing. The killing activity of the red thyme EO was compared with that of fluconazole, caspofungin and anidulafungin, some of the most used drugs in the prophylaxis and treatment of candidiasis. The results showed that the red thyme essential oil significantly stimulates the killing of intracellular C. albicans and C. krusei by PMN than essential oil-free controls, with percentages of killing similar (for C. albicans) or higher (for C. krusei) to those observed with fluconazole andanidulafungin. Conversely, the systems containing caspofungin further inhibit the survival of $C$. albicans with fungicidal activity superior to that of the essential oil and fluconazole.

To highlight if oil action was directed more on the yeast or the phagocyte, the extracellular killing of C. albicans by the oil in the absence of PMN was also evaluated.

In the absence of PMN, the essential oil activity, comparable to that of caspofungin, but higher than anidulafungin, was only fungistatic, suggesting that the increased intracellular killing by PMN could be attributable to a positive interaction between essential oil and phagocytes, as observed with conventional drugs.

Since the use of essential oils is attractive and the data obtained confirm their potential application, and encourage adequately controlled and randomized clinical investigations, including different screening methods and action mechanisms studies. It is desirable that the various experimental and clinical researches continue to improve the directions, methods and limitations of these therapeutic agents.

\section{Competing Interests}

The author declare that she has no competing interests.

\section{References}

1. Ciorba V, Odone A, Veronesi L, Pasquarella C, Signorelli C (2015) Antibiotic resistance as a major public health concern: epidemiology and economic impact. Ann lg 27: 562-579.
2. Wiederhold NP, Patterson TF (2015) What's new in antifungals: an update on the in-vitro activity and in-vivo efficacy of new and investigational antifungal agents. Curr Opin Infect Dis 28: 539-545

3. Tullio V, Nostro A, Mandras N, Dugo P, Banche G, et al. (2007) Antifungal activity of essential oils against filamentous fungi determined by broth microdilution and vapour contact methods. J Appl Microbiol 102: 15441550.

4. Tullio V, Mandras N, Scalas D, Allizond V, Banche G, et al. (2010) Synergy of caspofungin with human polymorphonuclear granulocytes for killing Candida albicans. Antimicrob Agents Chemother 54: 3964-3966.

5. Tullio V, Mandras N, Allizond V, Nostro A, Roana J, et al. (2012) Positive interaction of thyme (red) essential oil with human polymorphonuclear granulocytes in eradicating intracellular Candida albicans. Planta Med 78: 1633-1635. 